\title{
O PAPEL DO COMPARTILHAMENTO DO CONHECIMENTO A PARTIR DA GESTÃO DO CONHECIMENTO
}

\section{ARTIGO ORIGINAL}

REIS, Ingrid Weingärtner ${ }^{1}$, CAZARINI, Edson Walmir ${ }^{2}$

REIS, Ingrid Weingärtner. CAZARINI, Edson Walmir. O papel do compartilhamento do conhecimento a partir da gestão do conhecimento. Revista Científica Multidisciplinar Núcleo do Conhecimento. Ano 06, Ed. 06, Vol. 10, pp. 136-160. Junho de 2021. ISSN: 2448-0959, Link de acesso:

\section{https://www.nucleodoconhecimento.com.br/administracao/papel-do-} compartilhamento,

DOI: 10.32749/nucleodoconhecimento.com.br/administracao/papel-do-compartilhamento

\section{RESUMO}

$\mathrm{Na}$ circunstância organizacional a relevância do conhecimento é assunto sobre o qual não há mais debates. $O$ conhecimento tem dever estratégico para a criação de produtos e trabalhos ou que respondam às contínuas alterações de necessidades e vontades dos clientes. Este conhecimento como tal, não se encontra nas instituições,

\footnotetext{
${ }^{1}$ Mestre em Ciências no Programa de Engenharia de Produção da USP São Carlos, na área de conhecimento de Economia, Organizações e Gestão do Conhecimento e Aprendizagem Organizacional. Especialista em gestão organizacional de acordo com - Modelo de Gestão da Excelência da Fundação Nacional da Qualidade pelo SENAI/SC e também em metodologias de ensino a distância pelo Centro Universitário Claretiano. Graduada em Tecnologia em Gestão de Tecnologia da Informação pela Universidade do Sul de Santa Catarina.
}

\section{Orientador.}

RC: 88829

Disponível em: https://www.nucleodoconhecimento.com.br/administracao/papel-docompartilhamento 
mas sim é parcela dos indivíduos, das pessoas que as constituem. Ainda que aparente algo claro, o translado do conhecimento do indivíduo à organização é, na realidade, um desafio. Isto porque o conhecimento das pessoas não necessariamente está alinhado às necessidades da organização. Buscou-se com essa pesquisa identificar que recursos ou estratégias podem ser utilizadas para desenvolver atos de compartilhamento, baseados nos procedimentos de formação do conhecimento. Procurou, da mesma forma, explicar a divergência teórica que existe entre transferência e distribuição do conhecimento, demonstrações diversas vezes confundidas na ação. Sendo a distribuição do conhecimento um ato do indivíduo dentro das organizações. Foi fundamental conhecer as importantes barreiras que impossibilitam tais atos. Estes obstáculos podem estar relacionados ao modo de se comportar do próprio do sujeito ou acontecer em âmbito organizacional. Para a execução deste estudo, adotou-se como método a pesquisa-ação, que tem como característica principal a atuação dos indivíduos envolvidos no estudo. Assim, a partir da metodologia de pesquisa desenvolvida, se pode identificar barreiras importantes, tanto individuais como organizacionais que afetam o compartilhamento do conhecimento.

Palavras-chave: Gestão do conhecimento, Compartilhamento do conhecimento, Barreiras individuais, Barreiras organizacionais.

\section{INTRODUÇÃO}

O importante papel do conhecimento no desempenho das organizações pode ser considerado como consenso, uma vez que diversas disciplinas vêm tratando deste tema nos últimos anos. A busca pela diferenciação no mercado e pela inovação tem levado os empresários a investirem nestes assuntos. A forma como as organizações adquirem, geram e partilham o conhecimento é parte da possível resposta ao cenário que ora se vive. Este processo de criação do conhecimento é realizado por meio da conversação entre as pessoas, da troca de informações e experiência, em um 
ambiente em que o relacionamento entre elas é estimulado, fomentando a colaboração e a confiança.

Entretanto, é necessário considerar que além dos mecanismos institucionais de como se pode fazer esta gestão, há uma peça-chave nesta discussão que é o sujeito que faz parte da organização. O conhecimento é do sujeito, não da organização. Portanto, é necessário realizar a discussão sobre a gestão do conhecimento a partir da perspectiva de como extrair ou melhor aproveitar este conhecimento individual.

Adotando como paradigma a visão oriental de que o sujeito que aprende deve apropriar-se do objeto de aprendizado, busca-se identificar com esta pesquisa qual a importância do compartilhamento para a gestão do conhecimento. Que recursos ou estratégias podem apoiar as ações de compartilhamento, que relação se pode estabelecer entre estes e as fases do processo de criação do conhecimento SECI (TAKEUCHI e NONAKA, 2008) e, finalmente, que barreiras podem impactar em todo este processo.

Considerando a importância que tem no processo de criação do conhecimento, será essencial compreender como o compartilhamento do conhecimento acontece nas organizações, que elementos, que ações podem ser identificadas, tratadas e melhoradas visando a Gestão do Conhecimento.

Para realizar esta pesquisa, adota-se como metodologia a Pesquisa-ação, onde se considera fundamental a participação das pessoas envolvidas, desde o momento do planejamento até a demonstração de resultados. A participação é uma forma de realizar a prática de relacionamento social onde as partes envolvidas possuem conhecimentos que, ainda que sejam diferentes, podem ser integrados. Toda pessoa que é capaz de reconhecer e avaliar sua experiência é capaz de produzir conhecimento (TENÓRIO, 1990; THIOLLENT, 1996; GIL, 2008; GILBERTONI, 2012; SAMPIERI; COLLADO; LUCIO, 2006).

RC: 88829

Disponível em: https://www.nucleodoconhecimento.com.br/administracao/papel-docompartilhamento 
Este estudo será realizado em uma Instituição de Ensino Superior Privada, localizada em país Andino, envolvendo pessoal de nível diretivo e equipe técnico administrativa que gerencia processos de serviços acadêmicos, de apoio pedagógico e extensão universitária. Serão considerados processos tradicionais que estão sendo estruturados e formalizados neste momento histórico da Instituição, ou seja, existem, porém informalmente. Compreende-se que os conhecimentos e atividades realizados estão isolados em pessoas ou grupos de pessoas separadas por departamentos.

Será realizada pesquisa bibliográfica para compreender os fundamentos das ações do compartilhamento de conhecimento bem como conhecer quais as principais barreiras identificadas na atualidade.

Como ferramentas metodológicas para o desenvolvimento da pesquisa serão aplicadas a observação participativa, entrevista focalizada (GIL, 2008) e aplicação de pesquisa.

Como elementos estruturantes para a pesquisa-ação, na realização da observação serão consideradas as seguintes dimensões:

Quadro 1 - Dimensões da observação participativa.

\begin{tabular}{|l|l|l|}
\hline Dimensão & Definição & $\begin{array}{l}\text { Aplicação na pesquisa-ação } \\
\text { Universidade Técnica Particular de Loja - } \\
\text { UTPL - localizada em 84 cidades - } \\
\text { Equador. }\end{array}$ \\
\hline Atores & O lugar físico & $\begin{array}{l}\text { Pireção de Operações, Direções } \\
\text { Acadêmicas, Diretores de Centros } \\
\text { Regionais e Sub-centros, equipe técnica e } \\
\text { funcional. }\end{array}$ \\
\hline
\end{tabular}

RC: 88829

Disponível em: https://www.nucleodoconhecimento.com.br/administracao/papel-docompartilhamento 


\begin{tabular}{|c|c|c|}
\hline Atividades & $\begin{array}{l}\text { O conjunto de ações } \\
\text { que as pessoas } \\
\text { realizam }\end{array}$ & $\begin{array}{l}\text { Realização de processos de apoio à } \\
\text { gestão acadêmica, pedagógica e de } \\
\text { extensão universitária. }\end{array}$ \\
\hline Objetos & $\begin{array}{l}\text { As coisas físicas que } \\
\text { fazem parte da } \\
\text { realidade observada }\end{array}$ & $\begin{array}{l}\text { Uso de recursos e aplicação de } \\
\text { estratégias para o compartilhamento do } \\
\text { conhecimento. }\end{array}$ \\
\hline Atos & $\begin{array}{l}\text { Ações simples que as } \\
\text { pessoas fazem }\end{array}$ & $\begin{array}{l}\text { Definições de ações em cada processo; } \\
\text { planejamento conjunto de ações; } \\
\text { aplicação e controles. }\end{array}$ \\
\hline Eventos & $\begin{array}{ll}0 \text { conjunto de } \\
\text { atividades que as } \\
\text { pessoas executam }\end{array}$ & $\begin{array}{l}\text { Eventos definidos em calendário } \\
\text { institucional. }\end{array}$ \\
\hline Tempo & $\begin{array}{l}\text { A sequência que tem } \\
\text { lugar ao longo do tempo }\end{array}$ & $\begin{array}{l}\text { Os tempos são definidos em função do } \\
\text { planejamento das atividades e dos } \\
\text { calendários institucionais. }\end{array}$ \\
\hline Objetivos & $\begin{array}{l}\text { As coisas que as } \\
\text { pessoas estão tentando } \\
\text { realizar }\end{array}$ & $\begin{array}{l}\text { Alinhar e promover melhoras nos } \\
\text { resultados aos estudantes. }\end{array}$ \\
\hline Sentir & $\begin{array}{l}\text { As emoções sentidas e } \\
\text { expressadas. }\end{array}$ & $\begin{array}{l}\text { Insatisfação com resultados; frustração } \\
\text { com a perpetuação de mau desempenho. }\end{array}$ \\
\hline
\end{tabular}

Fonte: acervo pessoal.

\section{DESENVOLVIMENTO}

A Gestão do Conhecimento - O Conhecimento fundamental

A Gestão do Conhecimento faz parte do contexto global de um mundo sem fronteiras, onde os limites territoriais tradicionais não são mais tão relevantes para a gestão das organizações. De acordo com Ishikura (2008, p. 165) "As fronteiras nacionais 
significam menos atualmente do que antes, pois os clientes podem acessar informações e comprar produtos de todo o mundo".

Esta economia global, 'conectada' e fundada no conhecimento ganha força a cada geração, transferindo importância àquilo que é intangível, em detrimento do que é tangível e concreto. "O patrimônio indispensável para as empresas de hoje não é a fábrica e o equipamento, mas o conhecimento acumulado e as pessoas que o possuem" (ISHIKURA, 2008, p. 166). As empresas passam a competir no mercado com base em seus conhecimentos e ativos intelectuais (MASSARO, 2013; ISHIKURA, 2008).

No contexto das organizações, o conhecimento é definido como 'uma crença justificada' que potencializa a capacidade de uma empresa para uma ação eficaz. Esta definição é considerada mais apropriada do que uma definição filosófica do conhecimento, pois fornece uma descrição clara do conhecimento subjacente nas organizações (PEE, 2009; NONAKA, 1995). Já a Gestão do Conhecimento pode ser entendido como o processo de identificação e alavancagem do conhecimento coletivo nas organizações para ajudar a organização a se manter competitiva no mercado (TAKEUCHI; NONAKA, 1995; PEE, 2009).

O modelo de criação do conhecimento apresentado por Nonaka e Takeuchi (1995) considera fundamental apoiar-se no conhecimento tácito dos sujeitos como ponto de partida para criar um novo conhecimento organizacional. Este modelo apresenta a transição do conhecimento em duas dimensões: a dimensão ontológica, que é a transição do conhecimento desde o nível individual até o nível organizacional, e a dimensão epistemológica, que apresenta a transição entre o conhecimento tácito e o conhecimento explícito. Os autores denominaram este movimento de Espiral do Conhecimento. Ela representa as mudanças possíveis para a criação do conhecimento considerando 4 modos de conversão: Socialização, Externalização, Combinação e Internalização - SECI. 
Em cada um destes modos há a transição dos tipos do conhecimento, gerando um movimento de evolução. Na Socialização há a relação entre conhecimento tácito e tácito; na Extenalização, de tácito a explícito; na Combinação, de explícito a explícito; e na Internalização: de explícito a implícito.

O conhecimento tácito para Polonyi (1966) é a apreensão de toda uma determinada entidade, constituída pelas partes que a formam e por sua totalidade, ou seja, quando o observador compreende o objeto observado considerando suas partes e características - termo proximal - e sua integralidade - terma distal. Ainda que não detalhado, especificado e com este nível de consciência, o conhecimento tácito é o que o sujeito conhece por meio de sua percepção e intuição, não estando, portanto, formalizado ou sistematizado. Ainda de acordo com o mesmo autor, a percepção é individual, única, porque é influenciada pelas experiências pessoais.

Em estudos contemporâneos o conhecimento tácito dos sujeitos é um recurso diferenciado, que, se tornado consciente e espalhado por toda organização, leva à inovação e a solução de problemas concretos. O conhecimento tácito do sujeito é a base da criação do conhecimento organizacional (NONAKA e TAKEUCHI, 1995).

Por tanto, se pode compreender que no contexto organizacional que há o momento do conhecer sobre algo, logo uma toma de consciência e seu respectivo direcionamento ou aplicação e então a possibilidade de compartilhar este conhecimento.

\section{O Compartilhamento do Conhecimento}

De acordo com Castañeda, (2015), o compartilhamento de conhecimento é um comportamento fundamental na criação e aplicação do conhecimento, especialmente para as organizações. É um comportamento comum e essencial, identificado nas organizações que aprendem. Entretanto, este não é um comportamento automático, 
dependendo da vontade dos sujeitos envolvidos, de estímulos e de espaços conscientemente preparados para isso.

O compartilhamento do conhecimento surge como uma cadeia de eventos a partir da identificação do conhecimento chave necessário, das pessoas que necessitam desse conhecimento e dos recursos que podem apoiá-lo, tudo com base na interação social dos envolvidos. A comunicação é, por tanto, base para o compartilhamento do conhecimento. (LÓPEZ-FRESNO e SAVOLAINEM, 2015; ŠÁRKA, 2014).

Por suas próprias características, a comunicação não pode ser evitada, mas se pode atuar diretamente sobre ela para limitar ou melhorar as oportunidades de aprendizagem que são geradas a partir dela (ŠÁRKA, 2014).

Também se encontra que o compartilhamento do conhecimento se refere a prestação de informações sobre atividades e sobre conhecimentos para ajudar outras pessoas da organização, colaborando na resolução de problemas, desenvolvendo novas ideias, ou implementando políticas e procedimentos (WANG e NOE, 2010, GAÁL 2015 e IPE 2003).

A partir destes conceitos, se compreende que o compartilhamento do conhecimento é um ato humano, intencional, direcionado à criação de um conhecimento, que se utiliza de colaboração para esta construção e que pode ser identificado em cada um dos modos de conversão do conhecimento ou dentro de cada passo do processo de criação do conhecimento.

A partir da pesquisa realizada sobre a bibliografia especializada se identificam alguns elementos comuns em diversos autores que contribuem para que exista o compartilhamento do conhecimento. Estes elementos são tanto estruturais como elementos individuais. No quadro a seguir se organizam estes elementos. 
Quadro 2 - Elementos necessários ao compartilhamento do conhecimento.

\begin{tabular}{|l|l|}
\hline Elementos & Autores \\
\hline Confiança & $\begin{array}{l}\text { López-Fresno e Savolainen (2015), } \\
\text { Nesheim e Gressgardm (2014) e Šárka } \\
(2014)\end{array}$ \\
\hline $\begin{array}{l}\text { Espaço de relacionamentos formais } \\
\text { (reuniões) ou informais (redes sociais) }\end{array}$ & $\begin{array}{l}\text { López-Fresno e Savolainen (2015), } \\
\text { Wenger (2002), Caimo e Lomi (2015), } \\
\text { Gáal (2015) } \\
\text { Šárka (2014) }\end{array}$ \\
\hline $\begin{array}{l}\text { Valores compartilhados } \\
\text { média gestão }\end{array}$ & $\begin{array}{l}\text { Massaro et al (2014), Simons (1995) } \\
\text { Contação de histórias }\end{array}$ \\
\hline Presencialidade & $\begin{array}{l}\text { (2008) } \\
\text { Probst, Raub, e Romhardt (2000), } \\
\text { Nesheim e Gressgard (2014) }\end{array}$ \\
\hline Repetição de ações (bons exemplos) & Šárka (2014) \\
\hline Virtualidade & Nesheim e Gressgard (2014) \\
\hline
\end{tabular}

Fonte: acervo pessoal.

Diferença entre transferência de conhecimento e compartilhamento de conhecimento Apesar de que se encontra certa confusão entre ambas, a transferência de conhecimento não se confunde com as ações para o compartilhamento do conhecimento. Na transferência de conhecimento há um foco, um objetivo claro, e a unidirecionalidade da ação, enquanto no compartilhamento as ações podem se dar de forma não intencional em múltiplas direções sem um objetivo específico (BELLEFROID, 2012; IPE, 2003). 
Assim sendo, a transferência tem sempre uma intencionalidade e ocorre como resultado de um processo de pesquisa ou da geração de novos conhecimentos que serão aplicados em determinadas realidades. Por outra parte, o compartilhamento é meio, é maneira e etapa para a geração do conhecimento. São as ações corriqueiras de compartilhamento entre os sujeitos da organização que geram cada uma das etapas do processo SECI.

O compartilhamento é uma ação ou conjunto de ações que pode acontecer em qualquer etapa do processo de criação do conhecimento, utilizando-se de meios para o compartilhamento. O compartilhamento se dá por meio de uma relação dialógica e colaborativa desde os momentos iniciais do processo de criação do conhecimento, quando os sujeitos envolvidos no processo intercambiam seus conhecimentos tácitos ou mesmo os que são já explícitos, conscientes e já aplicados.

Barreiras à Criação do Conhecimento

Apesar da crescente importância das atividades de compartilhamento de conhecimento para a competitividade organizacional, várias barreiras dificultam o alcance de metas e melhora da competitividade no mercado global das organizações, afetando sua rentabilidade (CHONG, 2014; LINDSEY, 2006). A seguir são apresentadas as principais barreiras identificadas na bibliografia e pesquisas atuais.

São identificados dois níveis de barreias, as individuais e as barreiras organizacionais. As primeiras estão relacionadas ao comportamento e ações dos sujeitos e são identificadas. 
Quadro 3 - Relação das barreiras individuais e organizacionais à criação e compartilhamento do conhecimento e suas respectivas fontes.

\begin{tabular}{|c|c|c|}
\hline Categoria & Barreira & Fonte/origem \\
\hline Individual & $\begin{array}{l}\text { Falta ou baixa capacidade de } \\
\text { absorver e lidar com novas situações, } \\
\text { eventos, informações e contextos } \\
\text { diferentes; resistência interna. }\end{array}$ & $\begin{array}{l}\text { Kazuo Ichijo - Takeuchi } \\
\text { e Nonaka, Dixon } \\
(2000) \text {, Barson et al. } \\
(2000)\end{array}$ \\
\hline Individual & Meio em que vive. & $\begin{array}{l}\text { Kazuo Ichijo - Takeuchi } \\
\text { e Nonaka }\end{array}$ \\
\hline Individual & Experiências individuais. & $\begin{array}{l}\text { Kazuo Ichijo - Takeuchi } \\
\text { e Nonaka }\end{array}$ \\
\hline Individual & $\begin{array}{l}\text { Objetivos individuais em desacordo } \\
\text { com os objetivos organizacionais; } \\
\text { interesse individual. }\end{array}$ & $\begin{array}{l}\text { Kazuo Ichijo - Takeuchi } \\
\text { e Nonaka, Barson et al. } \\
\text { (2000) }\end{array}$ \\
\hline Individual & $\begin{array}{l}\text { Personalidade, timidez, medo ou } \\
\text { insegurança (não ter certeza de que o } \\
\text { conhecimento pessoal está correto); } \\
\text { Baixa confiança; dificuldade ou } \\
\text { incapacidade de perceber que o } \\
\text { conhecimento proposto agregará } \\
\text { valor. }\end{array}$ & $\begin{array}{l}\text { Kazuo Ichijo - Takeuchi } \\
\text { e Nonaka e Massaro, } \\
2014 \text { (p. } 121 \text { e 122), } \\
\text { Dixon (2000), Levina } \\
\text { (2001); Barson et al. } \\
(2000)\end{array}$ \\
\hline Individual & $\begin{array}{l}\text { Dificuldade ou incapacidade de } \\
\text { perceber que conhecimento se } \\
\text { encaixa no contexto atual. }\end{array}$ & Dixon (2000) \\
\hline Individual & Nível de educação formal. & $\begin{array}{l}\text { Nesheim e Gressgard, } \\
2014 \text {, p. } 29\end{array}$ \\
\hline
\end{tabular}

RC: 88829

Disponível em: https://www.nucleodoconhecimento.com.br/administracao/papel-docompartilhamento 


\begin{tabular}{|c|c|c|}
\hline Individual & $\begin{array}{l}\text { Experiência profissional; culturas } \\
\text { profissionais. }\end{array}$ & $\begin{array}{l}\text { Nesheim e Gressgard, } \\
2014 \text {, p. 29, Levina } \\
(2001)\end{array}$ \\
\hline Individual & Falta de vontade de escutar. & Levina (2001) \\
\hline Idividual & $\begin{array}{l}\text { Medo da exploração; medo de } \\
\text { contaminação, ou pensamento } \\
\text { proprietário. }\end{array}$ & Barson et al. (2000) \\
\hline Individual & $\begin{array}{l}\text { Ceticismo em relação ao } \\
\text { compartilhamento. }\end{array}$ & Barson et al. (2000) \\
\hline Individual & Tornar-se redundante. & Barson et al. (2000) \\
\hline ndividual & $\begin{array}{l}\text { Perda de poder; perda da } \\
\text { confidencialidade; responsabilidade } \\
\text { pessoal. }\end{array}$ & $\begin{array}{l}\text { Barson et al. (2000), } \\
\text { Cabrera \& Cabrera } \\
(2002)\end{array}$ \\
\hline Drganizacionais & $\begin{array}{l}\text { Necessidade de uma linguagem } \\
\text { legitimada; ausência de pistas } \\
\text { contextuais, } \\
\text { especializadas e metodologias. }\end{array}$ & $\begin{array}{l}\text { Kazuo Ichijo - Takeuchi } \\
\text { e Nonaka, Levina } \\
(2001)\end{array}$ \\
\hline Organizacionais & $\begin{array}{l}\text { Histórias organizacionais; } \\
\text { Paradigmas da empresa; fatores do } \\
\text { ambiente de operação, cultura e } \\
\text { crenças nacional, orientação local; } \\
\text { perda de memória, descontinuidade } \\
\text { de andamento em direção a metas, } \\
\text { cultura. }\end{array}$ & $\begin{array}{l}\text { Kazuo Ichijo - Takeuchi } \\
\text { e Nonaka; Okunoye } \\
\text { (2002), Levina (2001) }\end{array}$ \\
\hline Organizacionais & Procedimentos. & $\begin{array}{l}\text { Kazuo Ichijo - Takeuchi } \\
\text { e Nonaka }\end{array}$ \\
\hline rgan & $\begin{array}{l}\text { Tempo dedicado à realização do } \\
\text { trabalho mais significante do que ao }\end{array}$ & $\begin{array}{l}\text { Massaro, } 2014 \text { (p. } 121 \text { e } \\
\text { 122); Weiss (1999) }\end{array}$ \\
\hline
\end{tabular}




\begin{tabular}{|c|c|c|}
\hline & $\begin{array}{l}\text { Compartilhamento; limitações de } \\
\text { tempo. }\end{array}$ & \\
\hline Organizacionais & $\begin{array}{l}\text { O monitoramento das atividades de } \\
\text { trabalho inibe a criatividade e a } \\
\text { motivação para compartilhamento do } \\
\text { conhecimento; Controle de atividades } \\
\text { e excesso de medições; Cultura, } \\
\text { tecnologia, medição. }\end{array}$ & $\begin{array}{l}\text { Massaro, } 2014 \text { (p. } 121 \text { e } \\
\text { 122); KROGH, George } \\
\text { Von; ICHIJO, Kazuo; } \\
\text { NONAKA, Ikujiro, p. } \\
\text { 591; APQC, } 1996\end{array}$ \\
\hline Organizacionais & $\begin{array}{l}\text { No planejamento de projetos e } \\
\text { processos não se considera tempo } \\
\text { para a realização do } \\
\text { compartilhamento do conhecimento. }\end{array}$ & $\begin{array}{l}\text { Massaro, } 2014 \text { (p. } 121 \text { e } \\
\text { 122), McDermott \& } \\
\text { Odell (2001) }\end{array}$ \\
\hline Organizacionais & $\begin{array}{l}\text { Não há sistema de recompensa } \\
\text { identificado para esta atividade; falta } \\
\text { de recompensas, falta de } \\
\text { reconhecimento, falta de } \\
\text { reciprocidade; Contribuição, precisão, } \\
\text { reconhecimento. }\end{array}$ & $\begin{array}{l}\text { Massaro, } 2014 \text { (p. } 121 \text { e } \\
\text { 122); Weiss (1999); } \\
\text { Ellis (2001), McDermott } \\
\text { \& Odell (2001), Barson } \\
\text { et al. (2000), Cabrera \& } \\
\text { Cabrera (2002) }\end{array}$ \\
\hline Organizacionais & 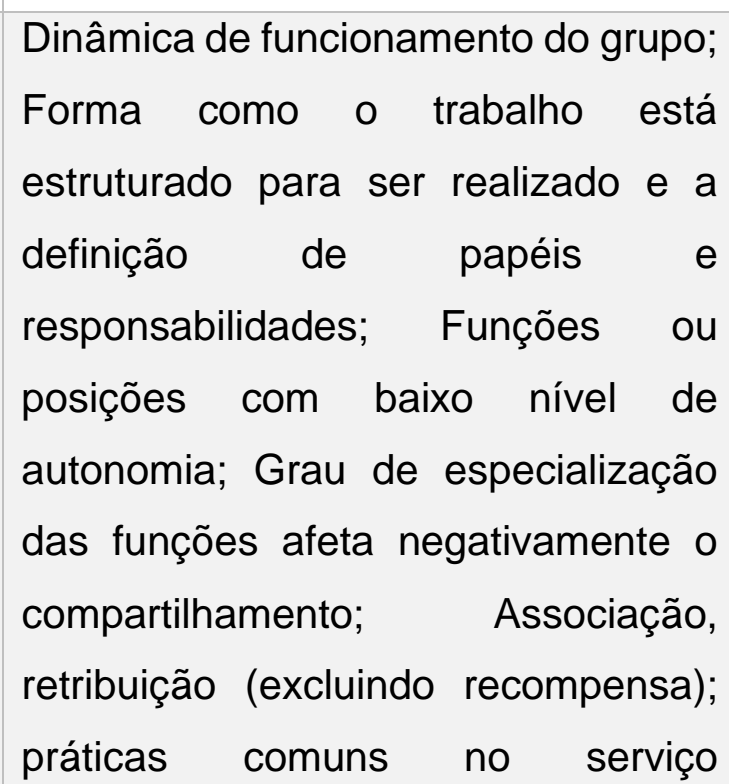 & $\begin{array}{l}\text { López-Fresno e } \\
\text { Savolainen, 2014, } \\
\text { Nesheim e Gressgard, } \\
\text { 2014, p. 29; Bock \& Kim } \\
\text { (2002); Weiss (1999), } \\
\text { Levina (2001); Dyer \& } \\
\text { Nobeoka (2000), } \\
\text { Cabrera \& Cabrera } \\
(2002)\end{array}$ \\
\hline
\end{tabular}

Disponível em: https://www.nucleodoconhecimento.com.br/administracao/papel-docompartilhamento 


\begin{tabular}{|c|c|c|}
\hline & $\begin{array}{l}\text { profissional; as diferenças na } \\
\text { unidade: subcultura, os objetivos da } \\
\text { unidade, as restrições de um } \\
\text { problema local; a prevenção de } \\
\text { aproveitadores, redução dos custos } \\
\text { de busca de conhecimento. }\end{array}$ & \\
\hline Organizacionais & Clima de trabalho. & $\begin{array}{l}\text { López-Fresno } \\
\text { Savolainen, } 2014\end{array}$ \\
\hline Organizacionais & Abordagem de gestão limitada. & $\begin{array}{l}\text { KROGH, George Von; } \\
\text { ICHIJO, } \quad \text { Kazuo; } \\
\text { NONAKA, Ikujiro }\end{array}$ \\
\hline Organizacionais & $\begin{array}{lcr}\text { Visão de que Gestão } & \text { do } \\
\text { Conhecimento } & \text { é fabricação } & \text { de } \\
\text { ferramentas } & \text { (ferramentas } & \text { e } \\
\text { instrumentos); } & \text { Gestão } & \text { do } \\
\text { Conhecimento } & \text { baseada } & \text { em } \\
\text { informações } & \text { detectáveis } & \text { e } \\
\text { quantificáveis. } & & \end{array}$ & $\begin{array}{l}\text { KROGH, George Von; } \\
\text { ICHIJO, } \quad \text { Kazuo; } \\
\text { NONAKA, Ikujiro }\end{array}$ \\
\hline Organizacionais & $\begin{array}{l}\text { Gestão do Conhecimento depende de } \\
\text { uma estrutura física e organizacional } \\
\text { (Knowledge officer). }\end{array}$ & $\begin{array}{l}\text { KROGH, George Von; } \\
\text { ICHIJO, } \quad \text { Kazuo; } \\
\text { NONAKA, Ikujiro }\end{array}$ \\
\hline Organizacionais & $\begin{array}{l}\text { A falta de um mecanismo de } \\
\text { compartilhamento de conhecimento; } \\
\text { Simplicidade, acesso, usabilidade, } \\
\text { motivação para participar; facilidade } \\
\text { de utilização. }\end{array}$ & $\begin{array}{l}\text { Fraser, Marcella \& } \\
\text { Middleton (2000); } \\
\text { Buckman Model (1998); } \\
\text { Hall (2001), McDermott } \\
\text { \& Odell (2001), Barson } \\
\text { et al. (2000), Barson et } \\
\text { al. (2000) }\end{array}$ \\
\hline Organizacionais & Rede que motiva a participação. & Dyer \& Nobeoka (2000) \\
\hline
\end{tabular}


Organizacionais Distância.

Organizacionais Personalidade, timidez, medo ou Kazuo Ichijo-Takeuchi insegurança (não ter certeza de que o e Nonaka e Massaro, conhecimento pessoal está correto); 2014 (p. 121 e 122), Baixa confiança; confiança; risco; Dixon (2002), Levina penalidade.
Barson et al. (2000) (2001); Barson et al. (2000)

Fonte: acervo pessoal.

\section{APLICAÇÃO E RESULTADOS}

A pesquisa foi realizada em uma Universidade que tem como principal característica a Educação a Distância. A Universidade, possui cerca de 40 mil estudantes, está localizada em um país Andino e neste contexto a educação a distância tem um papel muito importante, considerando a dificuldade de locomoção entre as cidades. A Universidade está localizada em 84 (oitenta e quatro) cidades do país, com suas unidades, atendendo a comunidade e o entorno social com ensino, pesquisa e ações de vinculação (extensão universitária).

Se há buscado identificar as principais barreiras individuais e organizacionais que influenciam ao compartilhamento do conhecimento entre as pessoas.

Como parte dos objetivos da pesquisa, está a identificação das principais barreiras encontradas no desenvolvimento da pesquisa-ação com o grupo de trabalho. Além da observação realizada ao longo da pesquisa, foi necessário validar com os envolvidos a percepção sobre as barreiras ao compartilhamento.

Para esta verificação tomou-se a relação geral das barreiras individuais e organizacionais e criaram-se perguntas que, organizadas, foram transformadas em

RC: 88829

Disponível em: https://www.nucleodoconhecimento.com.br/administracao/papel-docompartilhamento 
dois questionários, cujos objetivos eram de avaliar as barreiras individuais e organizacionais, a partir da perspectiva das pessoas.

Avaliação das Barreiras Individuais

As barreiras individuais são aquelas ligadas ao comportamento do indivíduo em relação à criação ou compartilhamento do conhecimento. Nesta etapa do trabalho do grupo foram selecionadas as 30 (trinta) pessoas mais influentes no desenvolvido das ações, localizadas em nível diretivo e tático. Desta população quatorze responderam ao questionário.

No Quadro a seguir estão apontados os resultados obtidos a partir da pesquisa realizada, com os índices obtidos a partir da avaliação, onde TD é a posição mais desfavorável e TA a mais favorável.

Quadro 4 - Perguntas aplicadas na pesquisa-ação e resultados da aplicação do questionário.

\begin{tabular}{|c|c|c|c|c|c|}
\hline Pergunta sobre Barreira Individual & TD & $D$ & $\begin{array}{ll}\mathrm{N} & \mathrm{D} / \mathrm{N} \\
\mathrm{A} & \end{array}$ & A & $\mathrm{TA}[3]$ \\
\hline $\begin{array}{l}\text { 1) Pessoalmente lido bem com } \\
\text { situações ou eventos novos }\end{array}$ & $7,14 \%$ & & $14,29 \%$ & $64,29 \%$ & $14,29 \%$ \\
\hline $\begin{array}{l}\text { 2) O conhecimento gerado pela } \\
\text { organização contribui para } \\
\text { minha formação pessoal }\end{array}$ & & & $21,43 \%$ & $42,86 \%$ & $35,71 \%$ \\
\hline $\begin{array}{l}\text { 3) Os objetivos da organização } \\
\text { coincidem com meus objetivos } \\
\text { pessoais e profissionais }\end{array}$ & & & $14,29 \%$ & $71,43 \%$ & $14,29 \%$ \\
\hline $\begin{array}{l}\text { 4) Meu conhecimento pessoal } \\
\text { contribui } \quad \text { para }\end{array}$ & & & & $50 \%$ & $50 \%$ \\
\hline
\end{tabular}

RC: 88829

Disponível em: https://www.nucleodoconhecimento.com.br/administracao/papel-docompartilhamento 


\begin{abstract}
desenvolvimento
da

organização

5) Meu conhecimento

profissional encaixa-se com o contexto da organização

6) Minha formação acadêmica é suficiente para agregar valor às discussões e à geração de conhecimento organizacional
\end{abstract}

$21,43 \% \quad 42,86 \% \quad 35,71 \%$

\begin{tabular}{|l|l|l|l|}
$14,29 \%$ & $21,43 \%$ & $57,14 \%$ & $7,14 \%$
\end{tabular}

7) Minha experiência

\begin{tabular}{|l|l|l|}
$7,14 \%$ & $64,29 \%$ & $28,57 \%$
\end{tabular} profissional agrega valor às discussões e à geração de conhecimento organizacional

8) Em geral, me sinto estimulado a escutar ou aprender sobre novos temas ou situações.

9) Considero importante e me sinto tranquilo quando comento minhas ideias com as pessoas do meu grupo de trabalho e também com outros grupos

10) Ainda que minhas ideias já \begin{tabular}{|l|l|l|l|}
\hline $7,14 \%$ & $14,29 \%$ & $57,14 \%$ & $21,42 \%$ \\
\hline
\end{tabular} tenham sido compartilhadas por outras pessoas

em determinadas discussões, considero importante manifestar-me sobre os temas.

11) Considero importante que o $14,29 \% \quad 21,43 \% \quad 28,57 \%$ conhecimento seja de alguma 


\section{forma centralizado para garantir} sua integridade

Fonte: acervo pessoal.

De forma geral, as respostas apresentam uma tendência positiva do comportamento das pessoas em relação às barreiras individuais. Elas se percebem abertas a novas ideias e a aprender novos assuntos, entendem que o perfil profissional está alinhado às necessidades da Universidade e que, individualmente, veem contribuindo para o crescimento e desenvolvimento organizacional.

Este resultado representa a percepção das pessoas em função do seu próprio comportamento em relação ao compartilhamento do conhecimento. Entretanto, no transcurso da observação realizada, esta foi uma tendência confirmada, ou seja, do ponto de vista individual, da ação do sujeito, identifica-se ações positivas. Talvez por tratar-se de um ambiente em que o conhecimento seja o objetivo e missão da organização, se tenha encontrado tal reação nas pessoas.

As barreiras individuais mais impactantes observadas durante a realização da pesquisa estavam relacionadas principalmente às experiências individuais e ao fato de as pessoas terem a consciência de que o que poderiam compartilhar realmente agregaria valor ao trabalho realizado. Foi possível identificar isto a partir das questões dois, quatro e nove, onde se buscava saber como as pessoas percebem o conhecimento dentro da organização e o seu próprio conhecimento em função do todo e se se sentem livres para manifestar este conhecimento individual. Da mesma forma nas questões três e cinco, onde as pessoas afirmaram uma identificação com os objetivos e contextos organizacionais, o que pode ser um fundamento ou explicação da liberdade de compartilhar anteriormente identificada. Também há uma elevada percepção a respeito das experiências profissionais individuais e de como estas contribuem no contexto organizacional (questão sete). 
As barreiras individuais mais relevantes identificadas por meio da pesquisa, questionário e observação, foram as que estão relacionadas no Quadro a seguir:

Quadro 5 - Relação das barreiras individuais identificadas na pesquisa como mais relevantes.

\section{Barreira individual}

Formação acadêmica

Relação conhecimento profissional individual e o contexto organizacional

Posicionamento individual ante novas situações

\section{Conhecimento gerado na organização para a formação individual}

\section{Estímulo a escutar e aprender}

\section{Centralização do conhecimento}

Fonte: acervo pessoal.

Avaliação das Barreiras Organizacionais

As barreiras organizacionais identificadas na pesquisa bibliográfica foram agrupadas da mesma forma que as individuais e serviram de base para elaboração do questionário aplicado ao mesmo universo de pessoas (envio do questionário a trinta pessoas e 15 respondentes).

As barreiras organizacionais são, possivelmente, as mais fáceis de serem identificadas e assim mesmo, as mais difíceis de serem tratadas, porque fazem parte dos sistemas próprios de cada organização e de sua cultura. A maior parte das barreiras aqui identificadas está nos fundamentos do modelo de gestão e na estrutura das organizações.

O Quadro a seguir apresenta as questões aplicados sobre as barreiras organizacionais e os resultados obtidos.

RC: 88829

Disponível em: https://www.nucleodoconhecimento.com.br/administracao/papel-docompartilhamento 
Quadro 6 - Perguntas aplicadas na pesquisa-ação e as respectivas respostas.

\begin{tabular}{|c|c|c|c|c|c|}
\hline $\begin{array}{l}\text { Pergunta sobre } \quad \text { Barreira } \\
\text { Organizacional }\end{array}$ & TD & $D$ & $\begin{array}{ll}N & D / N \\
A & \end{array}$ & $A$ & $\mathrm{TA}[4]$ \\
\hline $\begin{array}{l}\text { 1) Na organização, é possível } \\
\text { identificar um processo formal e } \\
\text { legítimo para compartilhar o } \\
\text { conhecimento. }\end{array}$ & $6,67 \%$ & $40,00 \%$ & $13,33 \%$ & $33,33 \%$ & $6,67 \%$ \\
\hline $\begin{array}{l}\text { 2) Os documentos que } \\
\text { formalizam processos, } \\
\text { procedimentos ou qualquer } \\
\text { outra instrução de trabalho } \\
\text { estão padronizados na } \\
\text { organização. }\end{array}$ & $13,33 \%$ & $26,67 \%$ & $26,67 \%$ & $20,00 \%$ & $13,33 \%$ \\
\hline $\begin{array}{l}\text { 3) Em geral, a operação e as } \\
\text { atividades diárias são tão } \\
\text { intensas que não conseguimos } \\
\text { compartilhar os conhecimentos } \\
\text { com nossa equipe ou com } \\
\text { outras equipes de trabalho. }\end{array}$ & & $6,67 \%$ & $6,67 \%$ & $53,33 \%$ & $33,33 \%$ \\
\hline $\begin{array}{l}\text { 4) Em geral, os controles } \\
\text { gerenciais sobre as atividades e } \\
\text { resultados não interferem na } \\
\text { criação ou no compartilhamento } \\
\text { do conhecimento. }\end{array}$ & $6,67 \%$ & $33,33 \%$ & $20,00 \%$ & $33,33 \%$ & $6,67 \%$ \\
\hline $\begin{array}{l}\text { 5) } 0 \text { planejamento e os } \\
\text { cronogramas de trabalho não } \\
\text { determinam tempos para o }\end{array}$ & & $20,00 \%$ & $6,67 \%$ & $40,00 \%$ & $33,33 \%$ \\
\hline
\end{tabular}


compartilhamento

de

informações e de conhecimento

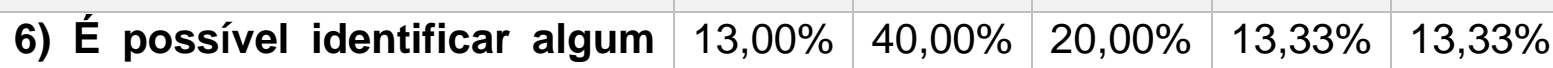
tipo de recompensa quando se trabalha no sentido de gerar e compartilhar o conhecimento

\begin{tabular}{|l|l|l|l|l|l|}
\hline 7$)$ A equipe ou grupo de pessoas & $6,67 \%$ & $26,67 \%$ & $33,33 \%$ & $6,67 \%$ & $26,67 \%$
\end{tabular} com as quais trabalho, em geral tem o hábito ou a predisposição de compartilhar

$\mathbf{0}$ conhecimento.

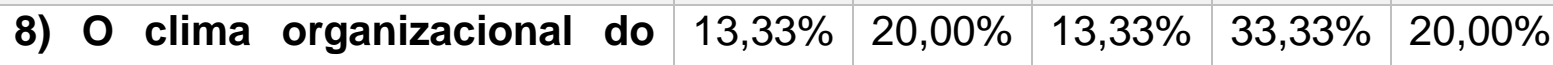
local onde trabalho é favorável ao compartilhamento de conhecimento

9) Os dirigentes aos quais me \begin{tabular}{|l|l|l|l}
\hline $13,33 \%$ & $6,67 \%$ & $40,00 \%$ & $40,00 \%$ \\
\hline
\end{tabular} reporto diretamente incentivam - compartilhamento do reconhecimento

10) Em geral os temas de informação e conhecimento são tratados como temas de tecnologia da informação

11) Em geral eu utilizo algum \begin{tabular}{|l|l|l}
$14,29 \%$ & $50,00 \%$ & $35,71 \%$
\end{tabular} meio tecnológico para compartilhar conhecimento

\begin{tabular}{|l|l|l|l|l|l|} 
12) Eu faço parte de uma rede o & $13,33 \%$ & $20,00 \%$ & $6,67 \%$ & $40,00 \%$ & $20,00 \%$
\end{tabular} de um grupo de pessoas com as quais posso compartilhar 0 


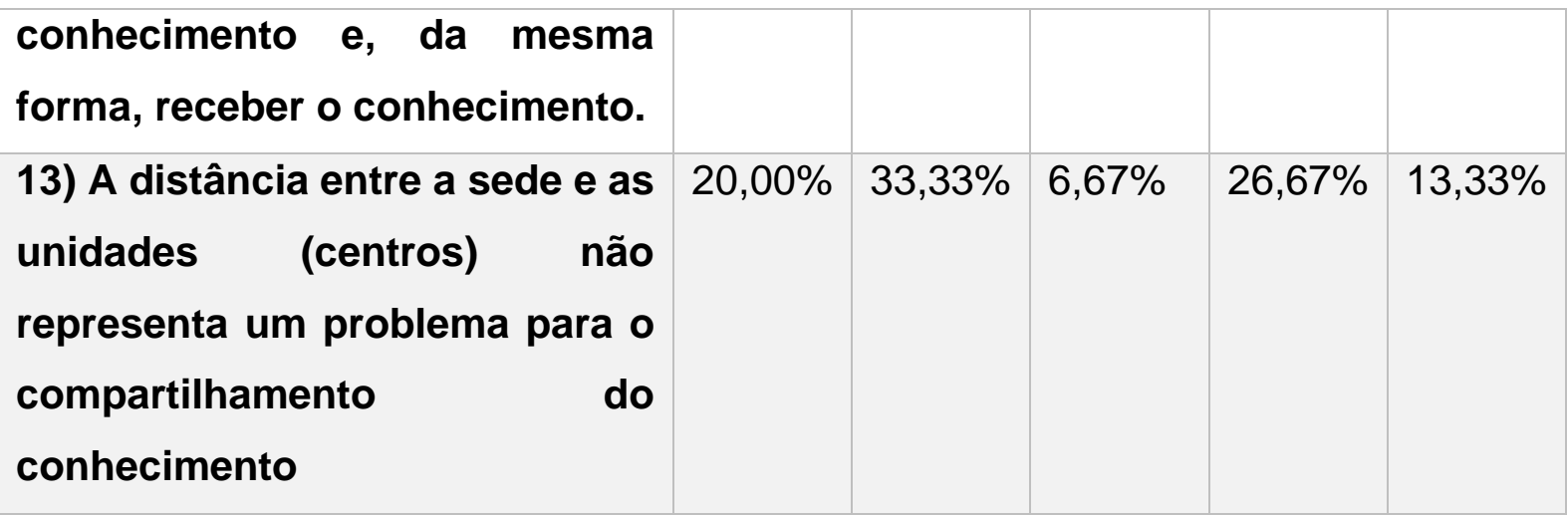

Fonte: acervo pessoal.

Com relação às barreiras organizacionais se percebe uma tendência nas respostas, apontando que não há consenso com relação aos esforços organizacionais em prol do compartilhamento do conhecimento. Verifica-se uma distribuição equânime das respostas entre os que estão totalmente de acordo e os que estão totalmente em desacordo com as questões apresentadas. Vale ressaltar que isso está baseado na formalização e consciência dos processos de criação de conhecimento dentro das organizações. Ainda que não estejam sistematizadas, existem ações institucionais de criação e que incentivam o compartilhamento do conhecimento.

De acordo com os resultados da aplicação do questionário a maioria dos respondentes não identifica a formalização dos conhecimentos dentro da organização. Entendem também que atividades voltadas à criação e ao compartilhamento do conhecimento não estão priorizadas nas atividades organizacionais. Durante o desenvolvimento da pesquisa-ação, na realização da observação, não foi possível identificar claramente os momentos de compartilhamento. Os planejamentos de trabalho não contemplam a avaliação das atividades realizadas. Com relação à questão sobre sistemas de recompensa (questão seis), a maioria dos respondentes não pode identificar formas de recompensa para ações que levem ao compartilhamento do conhecimento. Com relação a aspectos ambientais, a maioria das pessoas entende que o clima do ambiente onde estão inseridas é favorável ao compartilhamento do conhecimento. Que há incentivo dos responsáveis imediatos RC: 88829

Disponível em: https://www.nucleodoconhecimento.com.br/administracao/papel-docompartilhamento 
(80\%). Pela natureza da Instituição, pela vanguarda com que atuou no setor de ensino a distância, possui um conjunto grande de recursos de tecnologia que podem ser utilizados para o compartilhamento do conhecimento. Entretanto, apesar deste cenário, os respondentes do questionário entendem que o foco da Gestão do Conhecimento, do compartilhamento do conhecimento não está no uso de recursos tecnológicos. Mas que, de acordo com a questão onze, entendem a importância do uso destes recursos como apoio ao compartilhamento do conhecimento.

Quadro 7 - Relação das barreiras organizacionais identificadas na pesquisa como mais relevantes.

\section{Barreira Organizacionais}

Tempo: não priorização e ausência de planejamento de atividades para compartilhamento do conhecimento

Inexistência de processo formal de compartilhamento

\section{Ausência de um sistema de recompensa}

Visão vinculada entre gestão do conhecimento e tecnologia da informação

Fonte: acervo pessoal.

\section{CONSIDERAÇÕES FINAIS}

O compartilhamento do conhecimento é um processo social (LIN, 2015), portanto, mais do que qualquer ferramenta ou recurso, depende da iniciativa das pessoas e da intenção organizacional para que aconteça e seja efetivo.

Ao iniciar o planejamento do trabalho de pesquisa, foi realizado o levantamento dos recursos utilizados comumente. As categorias iniciais estavam direcionadas a coletar informações, controlar ações (execução de atividades e tarefas) e comunicar (base de conteúdos, blog, comunidade online, critérios de avaliação, organização de ideias e vídeo e comunicação).

RC: 88829

Disponível em: https://www.nucleodoconhecimento.com.br/administracao/papel-docompartilhamento 
Sendo fiel ao método indutivo, considerou-se que os resultados podem ser universalizados, todavia entende-se pertinente que a pesquisa seja replicada em outros contextos para aprofundamento e validação.

O grande desafio foi desenvolver a visão de que era necessário criar uma sistemática com finalidades claras e direcionadas à geração do conhecimento. Isto foi muito além do uso de recursos, pois se tratou de promover a possibilidade de colaborar na construção de algo. Nesta perspectiva, criar o ambiente para a realização do processo de criação do conhecimento envolveu a construção da confiança entre as pessoas, a definição de objetivos claros e compartilhados e a participação em todas as instancias de decisões.

A adoção do modelo SECl de Nonaka e Takeuchi (1995) para a criação do conhecimento orientou a realização do trabalho de pesquisa e respeitou o desenvolvimento das ações programadas.

Compreendido que o compartilhamento do conhecimento é um processo social, foi fundamental conhecer a partir da literatura os comportamentos individuais e organizacionais que em alguma medida poderiam impedir tais ações. Estes comportamentos negativos, ou barreiras, são facilmente reconhecidos no manejo organizacional. Em maior ou menor medida, estes comportamentos influenciam todo o processo de criação do conhecimento. As barreiras não surgem somente quando se está executando ações do processo de criação do conhecimento, logicamente. Elas são parte do comportamento organizacional. Entretanto, se pôde identificar que o fato de o trabalho ter sido realizado de forma colaborativa e aberto transformou o paradigma organizacional e que isto, de certa forma afetou a percepção das pessoas em relação às barreiras.

Considerando a avaliação feita sobre as barreiras individuais as pessoas sentem-se acolhidas institucionalmente. Percebem-se parte importante de um processo de construção organizacional, alimentando a Instituição e sendo alimentadas por ela.

RC: 88829

Disponível em: https://www.nucleodoconhecimento.com.br/administracao/papel-docompartilhamento 
Porém, quando a avaliação recai sob o aspecto organizacional, as mesmas pessoas apontam que não identificam um processo formal de recompensa ao compartilhamento do conhecimento.

Dentro do contexto de barreiras organizacionais, ainda não é possível identificar processos formais de compartilhamento do conhecimento. Logicamente existem dentro de todas as ações organizacionais, mas não faz parte do sistema de trabalho, não é percebido na íntegra e assim, não pode ser potencializado. Isto se pôde identificar pela falta de recompensa - como já mencionado, pela falta de formalização de cronograma de trabalho e pelos controles exigidos na execução das atividades. Entretanto, se identificou institucionalmente a predisposição das lideranças para o compartilhamento, assim como a percepção de um clima organizacional favorável.

O dever da distribuição do conhecimento, para o estudo realizado, foi definido a partir da identificação de seu conceito e aplicação em cada uma das etapas do processo de criação do conhecimento. Os recursos e estratégias pesquisados foram identificados como apoio ao compartilhamento do conhecimento. São meios que podem tornar o processo mais ágil, entretanto dependem necessariamente de uma intenção organizacional e individual.

As barreiras organizacionais e individuais atuam no sentido de tornar mais difícil ou inexistente o processo de compartilhamento do conhecimento. Entende-las auxiliará a compreender onde se deve atuar de forma a prevenir ou combatê-las.

Entende-se que existem muitas outras situações ou contextos em que se possa aplicar a mesma lógica de pesquisa, ou seja, avaliar as barreiras tanto em nível individual como em nível organizacional. 


\section{REFERÊNCIAS}

ALMEIDA, Maria da Conceição. Cenários da reorganização do conhecimento. Complexidade, saberes científicos, saberes da tradição. São Paulo: Ed. Livraria da Física, 2010. (Coleção Contextos da Ciência).

ALVES, Luiz Roberto. Ciência e consciência, conhecimento e liberdade. Estudos Avançados - USP, v. 26, n. 75, 2012. Disponível em: <http://www.revistas.usp.br/eav/article/view/39501>. Acesso em: 27 maio 2015.

ANTATAMULA, Vittal S.; KANUNGO, Shivraj. Modeling enablers for successful KM implementation. Journal of Knowledge Management, v. 14, n. 1, p. $100-113$, s/d.

BARSON, R. J., FOSTER, G. Struck, T. Ratchev, S. Pawar, K. Weber, F., et al. Inter - and - intra organizational barriers to sharing knowledge in the extended supply chain. Retrived December 2, 2002.

BELLEFROID, Bart. The new way of knowledge sharing. Utrecht University, 2012. 113 f. Master Thesis of Institute of Information and Computing Sciences, 2012.

BUTHELEZI, Mokateko; MKHIZE, Peter. Factors influencing quality of knowledge shared in software development community of practice. In: 11th International conference on intellectual capital, knowledge management and organisational learning ICICKM 2014. The University of Sydney Business School. Australia. Jim Rooney and Dr Vijaya Murthy University of Sydney. 2014.

CABRERA, A. CABRERA E. F. Knowledge-Sharing dilemmas. Organization Studies, 23 (5), $687-710.2002$.

CAIMO, Alberto; LOMI, Alessandro. Knowledge sharing in organizations: a Bayesian analysis of the role of reciprocity and formal structure. Journal of Management, v. 14, n. 2, p. $665-691,2015$,

RC: 88829

Disponível em: https://www.nucleodoconhecimento.com.br/administracao/papel-docompartilhamento 
CARRILLO, Javier. Managing Knowledge-based value system. Journal of Knowledge Management, p. $280-286$, v. 1, n. 4, 1998.

CASTAÑEDA, Delio; PARDO, Carlos; TOULSON. A Knowledge sharing instrument validation: broader perspective for global organizations. Eletronic Journal of Knowledge Management, v. 13, Issue 1, p. 3-12, 2015.

CHEN, Le; MOHAMED, Sherif. Contribution of knowledge management activities to organizationl business performance. Journal of Engineering, v. 6, n. 3, p. 269 - 285, 2008.

CHONG, Chin Wei; YUEN, Yee Yen; GAN, Geok Chew. Knowledge sharing of academic staff: A comparison between private and public universities in Malaysia. Library Review, v. 63, p. 203-223, 2014.

CRICELLI, Livio; GRIMALDI, Michele. Knowledge-based inter-organizational collaborations. Journal of Knowledge Management, v. 14., n. 3, p. 348 - 358, 2010.

CURVELLO, João José Azevedo; SCROFERNEKER, Cleusa Maria Andrade. A comunicação e as organizações como sistemas complexos: uma análise a partir das perspectivas de Niklas Luhmann e Edgar Morin. Revista da Associação Nacional dos Programas de Pós-Graduação em Comunicação, Brasília, v. 11, n. 3, set/dez. 2008.

DAVENPORT, Thomas H. Ecologia da Informação: porque só a tecnologia não basta para o sucesso na era da informação. São Paulo: Futura, 1998.

DIXON, Nancy M. Common Knowledge: How Companies Thrive by Sharing what they Know. Boston: Howard Business School, 2000.

FARIA, José Henrique de; MENEGHETTI, Francis Kanashiro. Dialética Negativa e a tradição epistemológica nos estudos organizacionais. Revista Organização e Sociedade UFBA, Salvador, v. 18, n. 56, p. 119-137, s/d.

RC: 88829

Disponível em: https://www.nucleodoconhecimento.com.br/administracao/papel-docompartilhamento 
GAÁL, Zoltán et al. Exploring the role of social media in knowledge sharing. The Electronic Journal of Knowledge Management, v. 13, Issue 3, p. 185-197, 2015.

GIL, Antonio Carlos. Métodos e técnicas de pesquisa social. São Paulo: Atlas, 2008.

GILBERTONI, D. A contribuição da pesquisa-ação na construção do conhecimento científico na Engenharia de Produção Brasileira. 2012. 194 f. Tese (Doutorado em Engenharia de Produção) - Programa de Pós-Graduação em Engenharia de Produção na Universidade Federal de São Carlos, São Carlos. 2012.

GUPTA, Babita et al. Knowledge management: practices and challenges. Industrial Management \& Data System, p. 17-21, 2000.

HART, Jane. Top 100 Tools for Learning 2015 GuideBook. Sine Loco: Center for Learning \& Performance Technologies, 2015.

HISLOP, Donald. Knowledge management as an ephemeral management fashion. Journal of Knowledge Management, v.14, n. 6, p. 779 - 790, 2010.

ICHIJO, Kazuo. Da administração à promoção do conhecimento. In: TAKEUCHI, Hirotaka; NONAKA, Ikujiro. Gestão do Conhecimento. Porto Alegre: Bookman, 2008.

IPE, Minu. Knowledge sharing in organizations: A conceptual framework. Human Resource Development Review, v. 2, n. 4, p. 337-359, 2003.

ISHIKURA, Yoko. Gestão do Conhecimento e concorrência global: a abordagem da Olympus à Gestão do Conhecimento global na indústria de fotografias digitais. In: TAKEUCHI, HIROTAKA; NONAKA, Ikujiro. Gestão do Conhecimento. Porto Alegre: Bookman, 2008.

IVES, Willian et al. Knowledge management: an emerging discipline with a long History. Journal of Knowledge Management, v.1, n. 4, p. 269-274, jun. 1998.

RC: 88829

Disponível em: https://www.nucleodoconhecimento.com.br/administracao/papel-docompartilhamento 
KAZEMI, Mostafa; ALLAHYARI, Mara Zafar. Defining a knowledge management conceptual modal by using MADM. Journal fo Knowledge Management, v. 14, n. 6, p. 872-890, 2010.

KROGH, George Von; ICHIJO, Kazuo; NONAKA, Ikujiro. Enabling Knowledge Creation. New York: Oxford University Press, 2000.

LEE, Maria R.; LAN, Yi-Chen. Toward a unified Knowledge management model for SMEs. Expert Systems with Applications, 38, p. 729-735, 2011.

LEHTONEN, Miika. Communicating competence through pechakucha presentations. Journal of Business Comunication, v. 48, n. 4, oct. 2011.

LEVINA, N. Sharing Knowledge in Hetereogeneous Environments. Reflections: The SoL Journal, 2 (2), 32 - 42. 2001.

LIN, Sheng Wei; LO Yi-Shih. Mechanisms to motivate knowledge sharing: integrating the reward systems and social network perspective. Journal of Knowledge Management, v. 19, n. 2, p. 212-235, 2015.

LINDSEY, Keith L. Knowledge sharing barriers. In: SCHWARTZ, D. G. (Ed.). The encyclopedia of knowledge management. Sine Loco: Idea Group Publishers, 2006.

LOPEZ-FRESNO, Palmira; SAVOLAINEN, Taina. Working meetings: a tools for building or destroying trust in knowledge creation and sharing. Electronic Journal of Knowledge Management, v. 12, Issue 2, p. 137-143, 2014.

MASSARO, M.; PITTS M.; ZANIN F.; BARDY R. Knowlwdgw Sharing, Control mechanisms anda intellectua liabilities in knowledge-intensive firms. Electronic Journal of Knowledge Management, v. 12, p. 117-127, Issue 2, 2014.

MCDERMOTT, R. O'DELL, C. Overcoming Cultural Barriers to sharing knowledge. Journal of Knowledge Management, 5 (1), 76 - 85, 2001.

RC: 88829

Disponível em: https://www.nucleodoconhecimento.com.br/administracao/papel-docompartilhamento 
MITCHELL, Rebecca; BOYLE, Brendan. Knowledge creation measurement methods. Journal of Knowledge Management, v. 14, n. 1, p. 67-82, 2010.

MOORADIAN, Norman. Tacit Knowledge: philosophic roots and role in KM. Journal of Knowledge Management, v. 9, n. 6, p. 104-113, 2005.

MORAES, Caroline Teixeira et al. Proposta de modelo de organização inovadora baseado na aprendizagem organizacional. INGEPRO - Inovação, Gestão e Produção, v. 3, n. 3, p. 92-105, mar. 2011.

MOUSTAGHFIR, Karim; SCHIUMA, Giovanni. Knowledge, learning and innovation: research and perspectives. Journal of Knowledge Management. v. 17, n. 4, p. 495510, 2013.

NESHEIM, T. GRESSGARD, L. J. Knowledge sharing in a complex organizations: antecedents and safety effects. Safety Science, v. 62, p. 28-39, 2014.

NONAKA, Ikujiro. A Empresa Criadora de Conhecimento. In: TAKEUCHI, Hirotaka; NONAKA, Ikujiro. Gestão do Conhecimento. Porto Alegre: Bookman, 2008.

; TAKEUCHI, Hirotaka. The knowledge - creating company: How Japanese Companies Create the Dynamics of Innovation. New York: Oxford University Press, 1995.

ORZANO, A. J. et al. A Knowledge management model: Implications for enhancing quality in healthcare. Journal of the American Society for Information Science \& Technology, Malden, v. 59, n. 3, p. 489-505, feb. 2008.

PEE, L. G.; KANKANHALLI, A. A Model of organizational knowledge management maturity based on people, process, and technology. Journal of Information \& Knowledge Management, Hackensack, v.8, n.2, p.1-21, 2009. 
PERROTTI, Edoardo. Estrutura organizacional e Gestão do Conhecimento. Dissertação (Mestrado). Departamento de Administração da Escola de Administração, Economia e Contabilidade da Universidade de São Paulo. São Paulo, 2004.

POLANYI, Michael. The tacit dimension. New York: Doubleday \& Company, INC. 1966.

POSTMAN, Joel. Social Corp: Social media goes corporate. Berkeley: New Riders, 2009.

RANJBARFARD, Mina et al. Identifying knowledge management problems using a process-based method (a case study of process 137). Business process management journal, v. 19, n. 2, p. $263-291,2013$.

ROLAND, Karen. Creating a knowledge community: embedded professional practice. In: 11th International Conference on Intellectual Capital, Knowledge Management and Organisational Learning ICICKM 2014. The University of Sydney Business School. Australia. Jim Rooney and Dr Vijaya Murthy University of Sydney. 2014. 321 - 327.

SAMPIERI, Roberto Hernandéz; COLLADO, Carlos Fernandéz; LUCIO, Pilar Baptista. Metodología de la Investigación. México: McGraw-Hill Interamericana, 2006.

SARKA, Hudcová. Tools of internal communication from Knowledge transfer perspective. Journal of Competitiveness, v. 6, Issue 4, p. 50-62, 2014.

TAKEUCHI, Hirotaka; NONAKA, Ikujiro. Gestão do Conhecimento. Porto Alegre: Bookman, 2008.

TENÓRIO, Fernando Guilherme. O mito da participação. Revista de Administração Pública, v. 24, n 3, maio/julho 1990.

THIOLLENT, Michel. Metodologia da pesquisa-ação. 7 ed. São Paulo: Cortez; 1996.

RC: 88829

Disponível em: https://www.nucleodoconhecimento.com.br/administracao/papel-docompartilhamento 
TOP 100 TOOLS FOR LEARNING 2015. Homepage: 9th Annual Survey of Learning Tools. Disponível em: <http://c4lpt.co.uk/top100tools/>. Acesso em: 21 maio 2016.

TUNG, Hui-Ling; CHANG, Yu-Hsuan. Effects of empowering leadership on performance in management team Mediating effects of knowledge sharing and team cohesion. Journal of Chinese Human Resource Management, v. 2, n. 1, p. $43-60$, 2011.

VUORI, Vilma. Social media changing the competitive intelligence process: elicitation of employees competitive knowledge. 2011. $239 \mathrm{fls}$. Thesis for the degree of Doctor of Science in Technology. Tampere University of Technology. Finland, 2011.

WANG, S. NOE, R. A. Knowledge sharing: a review and direcctions for future research. Human Resource Management Review, v. 20, p. 115-131, 2010.

WEISS, L. Collection and Connection: The Anatomy of Knowledge Sharing in professional service firms. Organization Development Journal, 17 (4), 61-77. 1999.

WENGER, Etienne; McDERMOTT, Richard; e SNYDER, William. Cultivating communities os practice. Boston: Harvard Business School, 2002.

WU, Yue et al. Diagnosis for organizational knowledge creation: an ontological shift SECI model. Journal of Knowledge Management, v. 14, n. 6, p. 791-810, 2010.

\section{APÊNDICE - REFERÊNCIA DE NOTA DE RODAPÉ}

3. Onde TD: Totalmente em desacordo; D de acordo; N D/NA: nem em desacordo, nem de acordo; A: de acordo; e TA: totalmente de acordo.

4. Onde TD: Totalmente em desacordo; D de acordo; N D/NA: nem em desacordo, nem de acordo; A: de acordo; e TA: totalmente de acordo.

Enviado: Maio, 2021.

RC: 88829

Disponível em: https://www.nucleodoconhecimento.com.br/administracao/papel-docompartilhamento 
Aprovado: Junho, 2021.

RC: 88829

Disponível em: https://www.nucleodoconhecimento.com.br/administracao/papel-docompartilhamento 